\title{
SERVIR EN ARAGON. LOS CORREGIDORES DE BORJA EN EL SIGLO XVIII
}

\author{
Enrique GIMENEZ LOPEZ \\ Jesús PRADELLS NADAL \\ Universidad de Alicante
}

El 26 de octubre de 1783, en cumplimiento del Capítulo VI de la Real Cédula de 29 de marzo de aquél año, el corregidor de Borja, Máximo Terol y Domenech, antes de su traslado a su nuevo destino de Alcira, dejaba a su sucesor una descripción poco alentadora del estado en que se hallaba Borja y del carácter de sus habitantes:

"No debo omitir el lamentable estado en que se halla lo florido de la ciudad, registrándose en ella la tercera parte del caserío derruido como si fuesen efectos de una guerra", para añadir en otro momento,

“...todo proviene de lo poco cuidadosos que son sus naturales, y nada interesados en su bienestar"।.

Máximo Terol era uno de los cientos de letrados anónimos que fueron columna vertebral de la administración borbónica en Aragón. Si la mayoría de sus nombres no despiertan entusiasmo alguno, y en pocas ocasiones se les ve sobresalir y apenas sabemos otra cosa de ellos sino que vivieron, casi siempre en condiciones penosas, una aproximación a sus biografias y a su actuación más o menos silenciosa en uno de los corregimientos situados en los confines de la monarquía, ayudará sin duda a la mejor comprensión de quienes cotidianamente y a pie de obra de la actividad administrativa ejecutaban las grandes lineas de la política setecentista.

Como ámbito geográfico donde aconteció su actividad hemos seleccionado el corregimiento de Borja, en Aragón. Su situación en un antiguo territorio foral con fuerte implantación de la jurisdicción señorial, en la frontera con Castilla, y su condición de corregimiento de Letras, permite reunir los ingredientes suficientes para considerarlo como paradigmático de corregimiento al que llegan letrados poco fogueados en la carrera de varas, y que anhelan, todavía, llegar a ocupar corregimientos de pátina más lustrosa y mejor acomodo y sueldo.

Borja era uno de los más modestos corregimientos aragoneses, creado como premio a su fidelidad borbónica durante la Guerra de Sucesión, y desde su origen fue corregimiento

1 A.G.S. Gracia y Justicia leg 825 Relación jurada que D. Máximo Terol de Domenech, actual corregidor de la cindad de Borja, y provisto por S.M. para el de Alcira, forma para presentarla al Supremo Consejo de la Cámara Borja, 26 de oclubre de 1783 
de Letras ${ }^{2}$. De Ios 22 letrados que lo sirvieron entre 1708 y 1808, más de la mitad vivieron en Borja su primer contacto con tierras aragonesas: 15 de ellos procedían de otros destinos, generalmente alcaldías mayores castellanas, andaluzas, catalanas o valencianas, y Borja era para la mayoría el primer corregimiento de letras que servían. Aquellos otros siete letrados que, por el contrario, habían ocupado puestos en la administración territorial aragonesa, lo habían hecho en cinco casos como alcaldes mayores de alguno de los corregimientos aragoneses de Capa y Espada. Borja era, pues, un paso intermedio en la carrera de varas, y así se puso de manifiesto cuando el Real Decreto de 29 de marzo de 1783, que reformaba el sistema corregimental español ${ }^{3}$, situó al corregimiento de Borja entre los de segunda clase, con un salario fijo sobre los propios de 8.282 rls. y 12 mvds. y que con los emolumentos podía superar los 10.000 rls.

El primer corregidor que se hizo cargo del gobierno de Borja "respecto de haber V.M. mandado establecer en el Reino de Aragón el mismo Gobierno y Tribunales que en los de Castilla" fue el navarro José de Anoz, oidor hasta entonces de la Cámara de Comptos de Navarra, y elegido por su cercanía a Borja, no muy alejada de la frontera con el reino navarro. Si atendemos a las difíciles circunstancias militares del momento, su toma de posesión fue relativamente rápida por la condición proborbónica de la ciudad, pues lo hacía el 30 de mayo de 1708 en un ambiente considerado por el corregidor propicio para la dificil misión encomendada:

"...teniendo la complacencia de ver el gusto con que estos buenos vasallos de S.M. me han recibido, haciendo expresiones dignas de fidelidad".

Los hechos pronto dejarían en entredicho el optimismo de Anoz, quien tuvo que abandonar precipitadamente Borja el 10 de abril de 1710 tras la derrota borbónica en Zaragoza. Regresaría el 10 de abril de 1711 solicitando la percepción de los 400 ducados de sueldo que se le adeudaban por el año en que había estado alejado de su puesto ${ }^{6}$. En ese mismo año de 1711, José Anoz fue ascendido a oidor del Consejo de Navarra, siendo el único caso de un corregidor de Borja que logró alcalzar la magistratura en alguno de los tribunales de la Monarquia.

El número de aragoneses que sirvieron el corregimiento de Borja fue reducido. Que sepamos sólo Antonio Francisco Triviño, Jose Miguel Herrero, durante el reinado de Felipe V, Manuel Laredo en el de Carlos III, y Juan Ignacio de la Pardina, en el de Carlos IV, fue-

2 Enrique GIMENEZ LOPEZ: “La Nueva Planta de Aragón. Corregimientos y Corregidores en el reinado de Felipe V", en Argensola 101 (1988), pp. 9-49.

3 Real Decreto comunicando a el Consejo de la Cámara con inserción de otro dirigido a el Consejo Real ambos en 29 de marzo de 1783 por los cuales establece S.M. el método sucesivo de proveerse y servirse los corregimientos y alcaldias mayores de los Reinos de Castilla y Aragón, e Islas adiacentes.

4 A.H.N. Consejos leg. 18.011 La Cámara propone a VM sujetos para el corregimiento de la ciudad de Borja 11 de enero de 1708.

5 A.H.N. Consejos leg. 18001 Testimonio de la toma de posesión del Corregidor de Borja D. José Anoz 30 de mayo de 1708 .

6 A.H.N. Consejos leg. 18.011 Don José Anoz, corregidor de Borja, suplica se le de por la Cámara despacho o Carta orden para que pueda cobrar lo destinado a su paga 1711. 
ron aragoneses que alcanzaron este cargo. El resto tenían naturaleza muy diversa: eran mayoritariamente castellanos, pero también los había de otros territorios de la antigua corona aragonesa, como sucedía con los casos del catalán Juan Bautista Font (1773-1777), o del valenciano Máximo Terol y Domenech (1780-1783). Los aragoneses Triviño y Herrero tenían tras sí una bien probada fidelidad a la causa borbónica: Antonio Francisco Triviño, catedrático de Instituta y Código en la Universidad de Zaragoza, había ocupado en los momentos más difíciles de la contienda sucesoria el cargo de Asesor Militar del Reino de Aragón y una de las alcaldias mayores de Zaragoza, de donde había pasado a Borja como sustituto de Anoz, falleciendo en 1712, poco después de acceder al cargo; José Miguel Herrero, corregidor entre 1712 y 1715 , había sido el primer alcalde mayor de Alcañiz, pero en 1710 tuvo que abandonar precipitadamente la ciudad y acompañar al ejército borbónico en retirada hasta Valladolid tras la batalla de Zaragoza?. Hasta 1784 no hubo ningún otro letrado aragonés que ocupara el corregimiento de Borja, haciéndolo entonces Manuel Laredo, un abogado formado en la Universidad de Zaragoza, y cuya carrera había transcurrido en su totalidad en tierras de Aragón, siendo alcalde mayor de Calatayud (1765-1768), corregidor de Benabarre en dos ocasiones (1768-1772 y 1775-1779), alcalde mayor de Huesca (1772-1775), corregidor de Barbastro (1780-1783), hasta llegar a Borja en 1784 y, finalizado su mandato en 1789 , pasar a la alcaldia mayor "más antigua" de la ciudad de Zaragoza. Considerado en la Corte como hombre "de buen juicio y desinterés", Manuel Laredo fue un letrado que contó en todo momento con la confianza del Consejo y Cámara de Castilla, en especial del consejero Pedro Ric y Egea, que pese a haber nacido en Fonz, Lérida, tenía profundas raices en Aragón, ya que en Huesca había sido Colegial de San Vicente y catedrático de Decreto de aquella Universidad ${ }^{8}$. El último de los corregidores aragoneses que sirvieron en Borja fue Juan Ignacio de la Pardina, un modesto pero activo miembro del movimiento ilustrado aragonés. Nacido en Barbastro en 1755 era doctor en derecho civil y canónico por la Universidad de Zaragoza, y fue alcalde mayor de Alcañiz y de Daimiel, cuyos servicios había finalizado en 1802. Era socio de número de la Real Sociedad Económica de Amigos del Pais de Zaragoza, y en esa institución obtuvo una cátedra de derecho público y desarrolló una discreta actividad ${ }^{9}$.

Los siete letrados que antes de ocuparse del corregimiento de Borja habían tenido a su cargo responsabilidades administrativas en territorio aragonés suponían algo menos de una tercera parte del total. Además de los aragoneses ya mencionados - Triviño, Herrero,

7 A.H.N. Consejos leg. 18.011 La Cámara propone a V.M. personas para el corregimiento de Letras de la ciudad de Borja 27 de abril de 1712.

8 Pedro Ric y Egea informó, al menos, en dos ocasiones sobre Manuel Laredo. En la primera ocasión, fechada en abril de 1765 , la carrera de Laredo se encontraba en sus inicios y había sido propuesto en primer lugar por la Cámara: "No le conoce, pero tiene referencia de que cursó Leyes en la Universidad de Zaragoza donde mostró talento y aplicación muy a propósito para el encargo de administrar justicia", en AGS Gracia y Justicia leg. 159 Sujetos propuestos para la alcaldia mayor de Calatayud 1765. En la segunda ocasión, Ric lo calificó de letrado dotado "de buen juicio y mucho desinterés", en A.G.S. Gracia y Justicia leg. 159 Sujetos propuestos para el corregimiento de Benabarre 1768.

9 E1 2 de septiembre de 1801 fue propuesto por la Cámara en primer lugar. A.H.N. Consejos leg. 18.011 La Cámara propone a V.M. sujetos para la vara de Corregidor de Borja 1801. 
Laredo y Pardinas - otros habían ocupado alcaldías mayores en el mismo Reino o, como en el caso de Francisco Lozano, otro corregimiento de letras aragonés. En algún caso la experiencia era muy dilatada, como sucedía con Fernando Busto Aguilar, que fue alcalde mayor de Zaragoza entre 1715 y 1720, y se hizo cargo del corregimiento de Borja veintiseis años después que finalizara en la alcaldia de la capital, pero era más usual que Borja apareciera en un punto intermedio del cursus honorum del funcionario letrado. Fernando Busto, natural de Alcaraz, en Albacete, de donde era regidor, inició precisamente su carrera en la administración en la alcaldía zaragozana, tras trabajar en Madrid de pasante y pretender algún cargo, situación a la que retornó en 1720 cuando aquejado por una enfermedad, hubo de concluir sus servicios en Zaragoza. La vida cotidiana del pretendiente, a la espera de ser incluido por la Cámara o por el Consejo de Ordenes en alguna propuesta para alguna vara o corregimiento, no era fácil. Para sobrevivir económicamente y hacer méritos a los ojos de los Consejeros de Castilla que elaboraban los informes reservados, el pretendiente debía aceptar las comisiones que los distintos Consejos, especialmente el de Castilla, proveían para la investigación o resolución de casos puntuales. Busto Aguilar efectuó una comisión en Torrelaguna, en la Serrania madrileña, al parecer con éxito, lo que le valió ser propuesto en segundo lugar para el corregimiento de Barbastro en 1721, con un informe de Baltasar de Acevedo, fechado el 21 de julio de aquel año, que lo calificaba como "desinteresado y sirve con amor y celo" ${ }^{10}$. Si bien no logró el corregimiento de Barbastro, Busto fue encargado en ese año de la alcaldia de la Mesta correspondiente a la cuadrilla de Soria, y en octubre de 1722 fue propuesto en segundo lugar para Borja, nombramiento que recaería a la postre en Sanchez Chaves. Pese a obtener informes favorables, no logró el empleo de corregidor de Bujalance, cerca de Córdoba, en 1725, pero un año después fue destinado para el de Requena ${ }^{11}$. Cuando fue propuesto en agosto de 1736 nuevamente para Borja, Fernando Busto Aguilar contaba con la favorable opinión de los Consejeros de Castilla Juan José de Mutiloa y José de Castro. El primero afirmaba que era "excelente abogado, con mucha madurez y juicio, y en todas partes que ha servido se ha portado muy bien y con mucho acierto", mientras que el segundo indicaba que tenía "común opinión de buen proceder y talento, y es suficiente para cualquier encargo"12.

De los dos restantes corregidores que habían servido en Aragón, uno de ellos, Francisco Lozano, lo había hecho con anterioridad en Barbastro, tras haberse hecho cargo de las alcaldias mayores de Alfaro, en La Rioja, y Arévalo, y era persona de edad avanzada cuando

10 A.G.S. Gracia y Justicia leg 136 Sujetos propuestos por la Cámara para el corregimiento de letras de Barbastro 1721.

11 Fue propuesto en primer lugar para el Corregimiento de Requena en 1726. En sus informes reservados Rodrigo Cepeda señaló que: "ha servido muy bien sus empleos, y que es muy digno de éste y de otro cualquiera corregimiento"; y Francisco Velázquez Zapata: "Es sujeto de habilidad y de mucho juicio y prudencia que ha manifestado en los empleos que ha servido" en AGS Gracia y Justicia leg. 138 Sujetos propuestos por la Cámara para el Corregimiento de Requena 1726.

12 A.G.S. Gracia y Justicia leg. 144 Sujetos propuestos por la Cámara para el Corregimiento de Borja en Aragón 1736. 
llegó a Borja en 1760 para fallecer en la ciudad donde era corregidor trěs ãños más tarde ${ }^{13}$. Juan Francisco Venero Mendoza, el otro corregidor, había comenzado sus servicios como alcalde mayor de las Cinco Villas de Aragón, de donde fue corregidor interino durante cinco años, de donde pasó a ocuparse de dos alcaldias importantes: la criminal de la ciudad de Granada y la civil de Zaragoza por nombramiento del Intendente Nicolás Zorrilla de San Martín. Cuando tomó posesión como corregidor de Borja en 1747 eran más de 21 años los que figuraban en su relación de servicios, y su estancia en la ciudad aragonesa fue del agrado de las autoridades municipales que en 1750 , cumplido el preceptivo trienio, solicitaron se le prorrogase el mandato hasta 1753 , lo que fue concedido.

La mayoría de los letrados que acudieron hasta Borja para hacerse cargo del corregimiento no habían tenido experiencia alguna en la administración aragonesa y sus servicios se reducían a algunas alcaldías mayores y a corregimientos muy modestos. En los casos de Juan Bautista Font, Máximo Terol y Manuel Antonio Denia previamente habían ejercido diversas alcaldías mayores en Cataluña, Valencia o Baleares. Juan Bautista Font es el único corregidor de origen catalán con que contó Borja. Nacido en Tárrega, y con estudios en la Universidad de Valencia, se inició en la carrera en 1752 como alcalde mayor de su pueblo natal, que sirvió hasta $1763^{14}$. En octubre de 1760 y agosto de 1761 fue propuesto en tercer lugar para la alcaldia mayor de Huesca ${ }^{15}$ y para el corregimiento de Tarazona ${ }^{16}$, y en 1768 , tras intervenir en varias comisiones confiadas por la Audiencia de Cataluña, fue designado para alcalde mayor de Balaguer, tomando posesión de la vara el 11 de febrero de $1769^{17}$. Propuesto para el corregimiento de Borja en noviembre de 1772, llegó a la ciudad aragonesa en 1773. Máximo Terol y Domenech era valenciano, nacido en Muchamiel, una villa huertana en las proximidades de Alicante, y formado en la teoria jurídica en la Universidad de Alcalá, y en la práctica en la madrileña Junta de Práctica Civil y Eclesiástica entre 1750 y 1752. Su primer destino fue en 1753 en tierras valencianas, designado como alcalde mayor de Oliva por el Consejo, ya que la villa de señorio del duque de Gandia se encontraba entonces secuestrada. Gregorio Mayans lo calificó de "ladrón manifiesto" en una de sus cartas a Manuel de Roda, cuyo contenido testimonia la proclividad al cohecho de un gran

13 En 1749, once años antes de ser propuesto para el corregimiento de Borja, el Consejero de Castilla Diego Adorno y Dávila informó de su edad avanzada:" Es de bastante edad y de juicio algo tímido en sus resoluciones, y de mediana literatura...", en A.G.S. Gracia y Justicia leg. 150 La Cámara 13 de octubre de 1749 propone para el corregimiento de letras de la ciudad de Barbastro 1749.

14 A.G.S. leg. 152 Sujetos propuestos para la Alcaldia Mayor de Tárrega 1752.

15 A.H.N. Consejos leg. 18.018 La Cámara propone a V.M. sujetos para la alcaldia mayor de la ciudad de Huesca 31 de octubre de 1760.

16 A.H.N. Consejos leg. 18.020 La Cámara propone a V.M. sujetos para el corregimiento de la ciudad de Tarazona 22 de agosto de 1761.

17 A.H.N. Consejos leg. 18.540 Real Decreto designando Alcalde mayor de Balaguer 23 de octubre de 1768. 
número de corregidores y alcaldes mayores, cuyos sueldos eran muy escasos ${ }^{18}$. En 1757 pasó a administrar justicia a Cofrentes, señorio del conde de Benavente, y en 1765 fue destinado a Morella como alcade mayor del teniente coronel marqués de la Torre del Barco, su corregidor $^{19}$. En noviembre de 1768 fue propuesto en segundo lugar para la alcaldia mayor de Calatayud ${ }^{20}$. De Morella pasó a Cataluña en 1770 , haciéndose cargo de la alcaldia mayor de Camprodón ${ }^{21}$, en la frontera con Francia, y destacando en la persecución del contrabando. Alcalde mayor de Castellón en 1773 por renuncia del letrado designado, Pedro José de Guizaburuaga $^{22}$, alcanzó definitivamente un corregimiento al ser elegido para el de Borja en $1780^{23}$, donde su labor se centró en efectuar algunos plantíos de pinos, álamos y encinas, e intentar intervenir coactivamente contra la población más menesterosa de la ciudad, jornaleros y mujeres. Según Terol, los jornaleros abusaban de los propietarios por no trabajar las horas debidas, y solicitó permiso al Consejo de Castilla para aplicar duras medidas, lo que le fue expresamente denegado. En julio de 1781 también representó al Consejo "la pereza, ociosidad y horror de estas mujeres a la ocupación propia de su sexo", que no era otra que hilar para potenciar en Borja su manufactura pañera, y culpando a las instituciones benéficas de la holganza femenina:

"...haciéndolas perjudiciales y vagantes la limosna de los conventos y la caridad mal entendida de los particulares, estando llenos sus zaguanes de mozas y mujeres mal ropadas, criándose en las porterias y tornos de monjas"24.

El tercer letrado con experiencia en territorios de la antigua corona aragonesa era Manuel Antonio Denia, corregidor de Borja muy pocos meses, ya que tomó posesión en 1808, momentos antes de que se iniciara la Guerra de la Independencia. Natural de Cuenca,

18 "Está governada por un ladrón manifiesto, que es Máximo Terol, mozo de levíssimo juicio, que no contento de ir provocando a torpezas abominables a muchas doncellas, viudas i casadas, por los lugares sum getos a la governación, ha hecho burdel al mismo palacio de los condes por escándalo universal. Tiene puestas, como en almoneda pública, las sentencias, vendiéndolas descaradamente al que le da más. Las penas ordinarias de diez escudos, de diez reales o veinte, las sube a veinte o más libras, i las cobra amenzando con autos criminales", en Gregorio Mayans a M. Roda, 6 de diciembre de 1755. Cfr. Epistolario Mayans con Manuel de Roda y Conde de Aranda, estudio preliminar de Antonio Mestre Sanchis, Valencia 1990, p. 167.

19 Sobre el marqués de la Torre del Barco, vid. Enrique GIMENEZ LOPEZ: Militares en Valencia (1707-1808). Los instrumentos del poder borbónico entre la Nueva Planta y la crisis del Antiguo Régimen Alicanted 1990, pp. 88-89.

20 A.H.N. Consejos leg. 18.012 La Cámara propone a V.M. sujetos para la vara de Alcalde mayor de la Ciudad de Calatayud 16 de noviembre de 1768

21 A.H.N. Consejos leg. 18.540 Toma de posesión del Alcalde mayor de Camprodón 20 de junio de 1770 .

22 A.H.N. Consejos leg. 18.241 Relación de méritos y servicios de D. Máximo Terol y Domenech 10 de noviembre de 1773 .

23 A.H.N. Consejos leg. 18.011 La Cámara propone a V.M. sujetos para el corregimiento de letras de la ciudad de Borja 1780.

24 A.G.S. Gracia y Justicia leg. 825 Relación jurada que D. Máximo Terol de Domenech, actual corregidor de la ciudad de Borja, y provisto por S.M. para el de Alcira, forma para presentarla al Supremo Consejo de la Cámara Borja, 26 de octubre de 1783. 
y con cincuenta años cumplidos cuando llegó a Borja, Manuel Antonio Denia había estudiado en la Universidad de Gandia, en la que se había doctorado en Leyes, y se había ganado la vida en alcaldias mayores extremeñas dependientes del Consejo de Ordenes hasta que pudo pasar a Mallorca a hacerse cargo de la alcaldia mayor de la ciudad de Palma, y en ella se mantuvo hasta $1800^{25}$.

Los restantes corregidores de Borja presentaban unos rasgos en los que predominaba la modestia de su cursus honorum. Para Alonso Anaya (1716-1722), Juan José González (1741-1745) y Francisco Ruiz Albornoz (1753-1757), Borja fue su primer corregimiento tras servir en alcaldias mayores de distinta entidad. Alonso Anaya Espinosa, como otros casos ya mencionados, se había iniciado en la administración señorial ${ }^{26}$, hasta conseguir ser designado alcalde mayor de El Escorial. Su buen hacer en el Real Sitio le permitió ascender a corregidor de Borja con informes muy favorables de Juan Milán de Aragón, Mateo Pérez Galeote y García Pérez Araciel ${ }^{27}$. Juan José González, un abogado que ejercía en Granada y posteriormente en Madrid, opositor sin éxito a una relatoria del Consejo de Ordenes, comenzó a pretender en 1727 y a ejecutar diversos encargos comisionados por el Consejo de Castilla. Un año después lograba, a través del Consejo de Ordenes, la alcaldia mayor de Uclés y en 1736 la no menos modesta de El Toboso, donde puso fin a enfrentamientos entre distintas familias. Pese a poseer buenos informes y ser propuesto en 1740 para el corregimiento de Alcaraz ${ }^{28}$, la alcaldia mayor de Manzanares en $1741^{29}$, y en agosto de 1736 para el corregimiento de Borja ${ }^{30}$, no logró empleo hasta que en 1741 fue designado, sin que figurara en la terna, para este corregimiento aragonès. Fracisco Ruiz Albornoz (1753-1757) era otro de los tres corregidores de Borja que con anterioridad habían sido alcaldes mayores, en este caso de Jerez y Tobarra, donde había tenido problemas con actuaciones poco claras. El Consejero Diego Adorno informaba en 1748: "Es de corta literatura

25 En 1807 pretendió ocupar nuevamente la alcaldia mayor palmesana, aunque sin éxito. Vid. A.H.N. Consejos leg. 18.011 Pretendientes a la vara de la ciudad de Palma 1807.

26 Alonso Anaya estaba al servicio del marqués de Malpica, y en sus señorios de Toledo ejerció su jurisdicción.

27 Juan Milán informó el 2 de enero de 1716 que se hallaba ejerciendo la alcaldia de El Escorial "con mucho acierto y cabal desempeño"; Mateo Pérez Galeote, el 5 de enero de ese mismo año destacaba su "integridad, pureza y buen juicio", mientras que Garcia Pérez de Araciel señalaba que "ha procedido bien y tiene expedición". Vid. A.G.S. Gracia y Justicia leg. 133 Sujetos propuestos para el corregimiento de Borja 1716.

28 Propuesto 2 Corregimiento Alcaraz en 1740. Antonio Aguado: "Ha sido Alcalde Mayor de Uclés y también sirvió la vara del Toboso, que en esta mantuvo en paz aquel pueblo serenando las discordias que habia entre varias familias y manifestando así en ella como en la antecedente, justificación, conducta y prudencia, habiendo merecido del Consejo de Ordenes la aprobación de sus providencias") (Juan Jose Mutiloa: "Es de mediana literatura y habilidad, pero no de la mejor conducta de lo que resultaron algunas disensiones en el Toboso, en donde como queda dicho fue Alcalde mayor") en AGS Gracia y Justicia leg. 147 La Cámara 12 de diciembre de 1740 propone para el corregimiento de letras de la ciudad de Alcaraz 1740.

29 Propuesto 2 Alcalde Mayor Manzanares en 1741. Juan Francisco de la Cueva: "Es buen letrado y prudente") (Gabriel de Olmeda: "Es buen sujeto") en AGS Gracia y Justicia leg. 147 El Consejo de Ordenes 23 de febrero de 1741 propone para la vara de Alcalde Mayor de la villa de Manzanares 1741.

30 Propuesto 2 Corregidor Borja en 22-VIII-1736. en AGS Gracia y Justicia leg. 18.011 La Cámara propone a VM personas para el corregimiento de letras de la ciudad de Borja 1736. 
y notado de no mucha limpieza y de alguna codicia por lo que el Consejo lo multó en 200 ducados en un pleito de partición de bienes que vino por recurso"31.

En cuatro casos el letrado designado para Borja ya había sido con anterioridad corregidor de una localidad de características similares o inferiores a Borja. Manuel Juan de la Parra (1766-1769) había sido corregidor de las Siete Merindades de Castilla la Vieja desde 1754 después de servir una alcaldía de la Mesta y la de Alfaro, y ser propuesto en 1750 como alcalde mayor de Daroca ${ }^{32}$; Bernardino Sanchez Chaves (1723-1726) había ejecutado diversas comisiones y pesquisas, y servido varias alcaldias y corregimientos antes de pasar a Borja, como las de Uclés o el corregimiento de Reinosa ${ }^{33}$; Diego Luis Tovar y Castro (1731-1736) fue corregidor de Olmedo hasta 1729, siendo posteriormente consultado en tercer y segundo lugar para los corregimientos de Tarazona ${ }^{34}$ y Albarracín ${ }^{35}$, indicándose en los informes su "corta práctica y experiencia" 36 ". Resulta excepcional el caso de Manuel Prado Castellanos que llegó a corregidor de Borja a los setenta y seis años de edad, como colofón de una larga carrera, para fallecer en ese cargo en 1760. En Ia reconstrucción parcial que del cursus honorum de Prado Castellanos hemos realizado, encontramos su nombre al frente de las alcaldias mayores de Guadix-Baza, Jerez de la Frontera, Segovia, y de los corregimientos de Vivero - considerado uno de los más pobres de la carrera-, Requena, Molina-Atienza y Tordesillas, último que desempeñó entre 1753 y 1756 antes de ser nombrado para el de Borja sin que su nombre figurara en la terna confeccionada por la Cámara. Si no tan longevo como Manuel Prado, la figura de Antonio Pérez de Goiburu tiene alguna similitud con la de aquél, pues Pérez de Goiburu fue nombrado corregidor de Borja a los setenta y seis años, cincuenta y siete años después de que hubiera sido nombrado alcalde mayor de Atienza y haber servido a la administración en multitud de alcaldias y corregimientos, entre ellos el corregimiento de Albarracín entre 1708 y 1711. Sin embargo, Pérez de Goiburu renunció a la plaza de Borja por su avanzada edad y solicitó que la vacante recayera en su hijo José, lo que después de ser aceptado por resolución de 12 de septiembre de 1745 no tuvo efecto por motivos de salud:

31 A.G.S. leg. 150 La Cámara 19 de junio de 1748 propone para el corregimiento de Letras de la villa de Utiel 1748.

32 A.G.S. Gracia y Justicia leg .151 La Cámara 7 de septiembre de 1750 propone para la vara de de Alcalde Mayor de la ciudad de Daroca 1750.

33 En su informe sobre Sanchez Chaves, el Consejero Baltasar de Acevedo indicaba que :"Ha servido muchos corregimicntos y es hombre conocido. En tiempo de D. Francisco Ronquillo estuvo en Villarrobledo en una pesquisa en que procedió bien, y últimamente en la pesquisa de Agreda contra el corregidor en que procedió arregladamente y con acicrto", en A.G.S. Gracia y.Justicia leg. 136 Sujetos propuestos por la Cámara para et corregimiento de letras de Borja 1722.

34 A.H.N. Consejos leg. 18.020 La Cámara propone a V.M. personas para el corregimiento de Letras de la ciudad de Turazona, 19 de mayo de 1729.

35 A.G.S. leg. 140 Sujetos propuestos por la Cámara para el corregimiento de Alharracín 1 de octubre de 1720 .

36 Intome reservado de Tomás Femández Molinillo, en A.G.S. Gracia y Justicia leg. 140 thidem. 
"Los médicos lo tienen privado del manejo de libros, y por consiguiente el entrar en dependencia a! runa, por lo que se halla impósibilitado por ahora de poder ejercer este correg miento",37.

Pero las designaciones de hombres de edad avanzada, al final de su carrera, como Prado o Pérez Goiburu, no era la norma. Para la mayoría de los letrados que sirvieron en Borja este destino suponía un paso intermedio en su carrera, y así se aprecia si seguimos la trayectoria posterior de aquellos que lo ocuparon. Algunos sirvieron otros corregimientos aragoneses de mayor entidad, como en los casos de Venero de Mendoza, que fue corregidor de Barbastro entre 1753 y 1756, Alonso Anaya Espinosa, que lo fue de Albarracín entre 1729 y 1733, y Juan Bautista Font que, tras ser propuesto sin éxito para las alcaldías mayores de Daroca (1777) y Alicante (1778), fue corregidor de Tarazona entre 1784 y 1789. Ninguno alcanzó puestos relevantes en la administración y ni tan siquiera fueron propuestos, que sepamos, para la magistratura en alguna de las Audiencias de la Monarquía. Sólo en el caso de Manuel Juan de la Parra hemos encontrado una referencia en las que se señala, sin mayor precisión, que "ha sido propuesto para plaza en las Audiencias de Indias", debido quizá a su vinculación familiar con Simón Mozo de la Torre, Secretario del Consejo de Indias, cuñado suyo. El resto tuvieron una discreta continuidad en la carrera administrativa, en alcaldías mayores de cierto relieve, como Plasencia o Algeciras, desempeñadas por Juan José González en Ios años cincuenta, o en corregimientos como Alcira - que sirvió Máximo Terol entre 1783 y 1792 - Vélez-Málaga o Lorca, ocupados por Francisco Ruiz Albornoz en los años sesenta ${ }^{38}$, o Alcaraz, concedido a Juan José González en 1763, treinta y ocho años después de que iniciara su actividad como abogado en Granada.

\section{LOS PRETENDIENTES PROPUESTOS POR LA CAMARA DE CASTILLA}

Algunos de Ios propuestos por la Cámara de Castilla para Borja tenían ya experiencia en tierras aragonesas como corregidores o alcaldes mayores. José Vicente Alcaide había sido corregidor de Albarracín antes de que en diciembre de 1752 fuera propuesto en segundo lugar para el de Borja, y Juan Prat Colomer acababa de finalizar en 1807 su mandato en Tarazona antes de que la Cámara lo presentara en el segundo lugar de la terna. En tres ocasiones el contacto con la administración aragonesa se había producido en la alcaldia mayor de Calatayud, relativamente proxima a Borja; eran los casos de Juan Antonio Pérez Vargas, Juan Galdón Cabezuelo, y Juan Bautista Ruiz Delgado.

En otros casos los servicios en Aragón se habían producido con posterioridad, como sucede con Francisco Ruiz Gálvez, propuesto en trecer lugar en la terna de 1722 y que fue corregidor de Tarazona entre 1725 y 1729, y Andrés Angel Durán Gómez, propuesto en el mismo lugar en 1763, y que en 1772 fue nombrado corregidor de Barbastro.

37 A.G.S. leg. 149 Corregimiento de Borja 1745. José Pérez de Goiburu, nacido en 1702, había sido con anterioridad corregidor de Bayona, en Galicia, durante seis años.

38 Francisco Ruiz Albornoz fue nombrado en 1768 corregidor de Lorca tras haber servido el corregimiento de Vélez-Málaga. Vid. A.G.S. Gracia y Justicia leg. 159 Sujetos propuestos para el corregimiento de Lor'a 1768. 
Entre los propuestos había algunos aragoneses, si bien sólo conocemos el lugar de nacimiento del $25 \%$ de los letrados incluidos en las ternas. Diego Franco de Villalba, nacido en Belmonte, era sin duda el más sobresaliente de todos ellos: catedrático de la Universidad zaragozana y abogado en ejercicio en la capital, partidario de la causa borbónica, fue propuesto el 20 de julio de 1711 para el corregimiento de Borja ${ }^{39}$, pero pronto pasaría a servir una de las alcaldías mayores de Zaragoza y, desde 1714, se incorporaría a una de las alcaldías del Crimen de la Audiencia de Aragón en plaza reservada a "naturales", contando con el apoyo de Melchor de Macanaz que lo consideraba "hombre de méritos, de gran literatura y familia", gran conocedor de los fueros aragoneses, a los que dedicó su Fororum ac observantiarum Regni Aragomun Codex ${ }^{40}$, si bien su conocimiento de las leyes castellanas era inferior, lo que le impidió ser designado fiscal de la Chancilleria de Valladolid $^{41}$. Franco de Villalba de mantuvo como alcalde del crimen de la Audiencia aragonesa hasta 1723 , en que fue promovido a oidor del mismo tribunal ${ }^{42}$, con el informe muy favorable del presidente de la Chancilleria de Granada, Lucas Fernández de la Fuente:

"...digno y muy a propósito para el empleo. Lo conoció siendo ministro en Zaragoza donde entonces ejercía la abogacía, en que se distinguía y conciliaba especial estimación de todos los de la profesión, así por las defensas que hacía como por los papeles que escribía"43,

que no sólo eran de literatura jurídica, sino religiosa, como la hagiografia de Sor Inés de Jesús y Franco ${ }^{44}$, y un largo texto sobre la veneración a los Angeles Custodios ${ }^{45}$ fechado en 1740 , nueve años antes de su fallecimiento.

El otro aragonés propuesto era Manuel Martinez Lamac [o Lansac], nacido en Daroca en 1760, con estudios en la Universidad de Zaragoza y abogado en ejercicio desde 1789. Antes de ser propuesto en segundo lugar en septiembre de 1801 para el corregimiento de Borja $^{46}$, había ocupado la alcaldia mayor de las Encartaciones en Vizcaya.

39 A.H.N. Consejos leg. 18.011 La Cámara propone a V.M. personas para el corregimiento de letras de la ciudad de Borja 20 de julio de 1711.

40 Diego FRANCO DE VILLALBA: Fororum ac observantiarum Regni Aragomun Codex, sive enodata methodica compilatio Jure civili et canonico fulcita, legibus Castellae conciliata, atque omnigena eruditione contexta Caesaraugustae, Petrum Ximenez 172, 755 pp., con una segunda edición en dos volúmenes publicada también en Zaragoza en 1743.

41 A.G.S. leg. 133 Fiscalia de la Chancilleria de Valladolid, 1714.

42 Ya en 1721, en dos ocasiones, y en el mismo año de 1723, había sido propuesto para oidor de la Audiencia aragonesa, con buenos informes de los consejeros José de Castro, José Rodrigo y el regente de la misma audiencia, quienes destacaron sus conocimientos forenses. Vid. A.G.S. leg. 136 Sujetos propuestos por la Cámara para la plaza de Oidor vacante en la Audiencia de Aragón, 1721; y Ibídem, leg 137 Oidor Audiencia de Aragón 1723.

43 A.G.S. Gracia y Justicia leg. 137 Oidor Audiencia de Zaragoza 1723.

44 La heroyna religiosa Sor Inés de Jesús y Franco, cuya vida exemplar y esclarecidas virtudes describe y publica $D$. Zaragoza 1733, 204 pp.

45 Devota excitación para el incesante reconocimiento y continua gratitud, conque todos debemos corresponder y venerar a los gloriosísimos Santos Angeles, y especialmente a los Custodios... Zaragoza 1740.

46 A.H.N. Consejos leg. 18.011 La Cámara propone a V.M. sujetos para la vara de Corregidor de Letras de la ciudad de Borja 2 de septiembre de 1801. 
Gran parte de los propuestos para Borja tuvieron empleos modestos en la carrera y, por lo que sabemos, llevaron una existencia difícil. El caso más llamativo es el de Francisco Antonio Salgado Arias, un madrileño bachiller en leyes por Alcalá desde finales de 1704 y que tras trabajar de pasante durante cinco años para el Consejero de Guerra Andrés González Barcia, fue enviado a gobernar los señorios de Moguer y Barcarrota en situación de tenuta por el Consejo. En la reforma de Macanaz fue designado Teniente de Alcalde de Casa y Corte $^{47}$, y reformado al producirse la caida de Macanaz y la consiguiente contrarreforma de los Consejos en 1715, al igual que Manuel Correa Canseco ${ }^{48}$, otro de los propuestos para Borja en 1715. Comenzó entonces para Salgado la dura existencia del pretendiente: fue propuesto entre 1715 y 1718 , además de Borja, para los corregimientos de Orense, las Cuatro Villas de la Hoya de Málaga, Barbastro y Olmedo. En 1718, tras la conquista de Cerdeña y la muerte del oidor Juan Serrano Espejo, solicitó la plaza ${ }^{49}$, pero le fue concedida la de Alcalde del Crimen. Llegado a Barcelona con su mujer y sus cinco hijos el 12 de noviembre de 1718 para embarcarse, no lo pudo hacer por lo dificil de la situación militar. Regresó a Madrid y volvió a la condición de pretendiente: en julio de 1719 solicitó una de las vacantes existentes en la Audiencia de Cataluña por fallecimiento de los oidores Juan Antonio de Navas y Gregorio de $\mathrm{Matas}^{47}$, que no le fue concedida y, finalmente, pudo ocupar el corregimiento de Andújar ${ }^{51}$ en 1720. En 1726, lo encontramos de nuevo en la Corte en situación económica lastimosa. El camarista de Castilla Pascual de Villacampa describía su estado en estos inequívocos términos:

"...ni es decoro de S.M. ni del ministerio que se vea por las calles un ministro togado pidiendo limosna (...); se halla tan imposibilitado de medios como lo acredita su miserable e indecente porte ${ }^{51}$.

El caso de Salgado, que hemos descrito con detalle por ejemplarizar la figura del pretendiente que vive penosamente en la Corte, no era extraña sino usual entre los letrados que eran incluidos sin fortuna en las ternas que elaboraba la Cámara de Castilla: Juan

47 En el informe para la provisión de la Tenencia de Alcaldia de Casa y Corte, Melehor de Macanaz indicaba de su puño letra al referirse a Salgado Arias:"Que se le provea la plaza", en A.G.S. Gracia y Justicia leg 133 Sujetos propuestos para un Tenencia de Alcalde de Casa y Corte, 26 de julio de 1714. Tomó posesión de la plaza el 18 de agosto de ese mismo año.

48 Juan Milán de Aragón señalaba en su informe de 2 de enero de 1716 sobre Manuel Correa: "Fue reformado de su empleo de Teniente de Alcalde de Casa y Corte"; y Garcia Pérez de Araciel el 4 de enero de el mismo año señalaba:"habiendo venido a la Corte se le hizo en la Nueva Planta Teniente de Alcalde", en A.G.S. Gracia y Justicia leg. 133 Sujetos propuestos para el corregimiento de Borja, y también en A.H.N. Consejos leg. 18.011 La Cámara propone a V.M. personas para el corregimiento de letras de Borja, 17 de diciembre de 1715 .

49 A.G.S. leg. 134 Memorial de D. Francisco Salgado Arias pretendiendo ser oidor de la Audiencia de Cerdeña 1718 .

50 A.G.S. Gracia y Justicia leg. 134 Memorial de D. Francisco Salgado Arias pretendiendo una plaza de Oidor en la Audiencia de Cataluña 1719.

51 A.G.S. Gracia y Justicia leg. 135 Corregimiento de Letras de Andújar, 1720.

52 A.G.S. Gracia y Justicia leg. 138 Pascual de Villacampa sobre la pretensión de D. Francisco Antonio Salgado a la Alcaldia del Crimen de la Chancilleria de Granada, 17 de noviembre de 1726. 
Antonio Pérez Vargas, propuesto en enero de 1741 para Borja, solicitaba nuevamente esa plaza, junto a las de Orense, Alcaraz y Molina "por hallarse más de tres años sin ocupación y en pretensión en esta Corte" "53; y Juan Francisco Bernal, propuesto en tercer en 1757, había sido considerado por Cristobal Monsoriú siete años antes como "pobre y poco inclinado a trabajar" ${ }^{54}$. En su mayoría habían estado al servicio de casas nobiliarias como corregidores de señorios: Félix Yerro Pérez de Lara tuvo su primer trabajo en Albanchez, en Almeria; José de Avillón en el señorio valenciano de Chelva; Pablo Acosta estuvo al servicio del duque de Frias como corregidor de Villadiego; y Juan Troche fue alcalde en distintos señorios de los duques de Huéscar y Alba entre 1770 y 1776. Durante la primera mitad de siglo muchos de los propuestos para Borja fueron alcaldes mayores por designación de sus corregidores respectivos: Manuel Correa, tras sus estudios en Alcalá, fue alcalde mayor de Villaescusa de Haro y Plasencia entre 1704 y 1710; Francisco José Ruiz Gálvez, fue alcalde mayor de Ubeda primero, y de Córdoba después por nombramiento del corregidor Luis de Mergelina; Juan Antonio Pérez Vargas era Alcalde mayor de Calatayud por nombramiento del corregidor Luis Salcedo; Félix Yerro sirvió la alcaldia de Castellón de la Plana... etc.

Los destinos que alcanzaron a lo largo de su experiencia administrativa fueron modestos, a la altura del corregimiento de Borja al que optaron, sin éxito, en una determinada ocasión. Hemos logrado localizar doce distintos corregimientos de Letras no aragoneses en los que sirvieron letrados que fueron propuestos para el de Borja. Si utilizamos como criterio para fijar el nivel de categoria del corregimiento la distribución en tres clases establecida por Campomanes en 1783, y recordando que los incluidos en la primera clase eran de entrada, es decir, los más modestos y con asignaciones inferiores a los 12.000 reales anuales, y que Borja fue considerado corregimiento de segunda clase, obtenemos que ocho de esos doce corregimientos eran de la categoría inferior (Madrigal, Cuatro Villas de la Hoya de Málaga, Vivero, Bayona, Aranda-Sepúlveda, Olmedo, Jijona [en dos casos] y Santo Domingo de la Calzada), mientras que Tordesillas, en dos ocasiones, y MolinaAtienza, eran de segunda clase, y sólo Andújar, en Jaen, se encontraba entre los del nivel superior. Una evidencia más del escaso fuste que en la carrera de varas tuvieron los letrados que la Cámara barajó para cubrir el gobierno de este corregimiento pequeño y periférico.

53 A.G.S. Gracia y Justicia leg .148 Memorial de Juan Antonio Pérez Vargas y Maldonado, 1742.

54 A.G.S. Gracia y Justicia leg. 151 La Cámara 2 de septiembre de 1750, propone para la vara de Alcalde Mayor de la villa de Alcira 1750. 